\title{
TIMP3 attenuates cerebral ischemia/reperfusion-induced apoptosis and oxidative stress in neurocytes by regulating the AKT pathway
}

\author{
LINGLEI MENG ${ }^{1}$, YONGTING ZHANG ${ }^{2}$, DEMAO LI ${ }^{3}$, XINFANG SHANG ${ }^{1}$, \\ XUEJIA HAO $^{1}, \mathrm{XIN} \mathrm{CHEN}^{4}$ and FENGXIAO GAO ${ }^{1}$ \\ ${ }^{1}$ Department of Imaging, Xingtai People's Hospital; ${ }^{2}$ Department of Imaging, Xingtai Orthopedic Hospital; \\ Departments of ${ }^{3}$ Cardiothoracic Surgery and ${ }^{4}$ Neurology, Xingtai People's Hospital, Xingtai, Hebei 054001, P.R. China
}

Received May 13, 2020; Accepted May 26, 2021

DOI: 10.3892/etm.2021.10405

\begin{abstract}
Ischemic stroke seriously threatens human health and creates a large social burden. The present study investigated whether tissue inhibitor of metalloproteinases-3 (TIMP3) prevented cerebral ischemia/reperfusion (I/R), with the aim to explore the underlying mechanism. A transient middle cerebral artery occlusion model was conducted in mice, and oxygen glucose deprivation and reoxygenation (OGD/R) was investigated in PC12 cells to mimic cerebral ischemia-reperfusion injury (CIRI). Western blotting was used to determine the expression of TIMP3, Bax, Bcl-2 and AKT. TUNEL was used to detect apoptosis in cerebral tissues or cultured PC12 cells. Expression levels of reactive oxygen species (ROS), superoxide dismutase (SOD) and malondialdehyde (MDA) were detected to reveal oxidative stress. The results demonstrated that TIMP3 expression was significantly decreased after $\mathrm{I} / \mathrm{R}$ in vivo or $\mathrm{OGD} / \mathrm{R}$ in vitro, and the number of TUNEL-positive cells was reduced by the overexpression of TIMP3. The attenuation of $\mathrm{Bax} / \mathrm{Bcl}-2$ ratio in OGD/R-induced PC12 cells suppressed the expression levels of ROS and MDA; while also elevating SOD activity in the OGD/R-induced neurocytes in vitro. In addition, TIMP3-overexpression reversed the downregulation of phosphorylated-AKT (Thr308 and Ser473) in OGD/R-treated PC12 cells. However, the anti-apoptotic and anti-oxidative stress roles of TIMP3 in OGD/R-induced PC12 cells were partially abolished after treatment with the AKT inhibitor, AZD5363. Overall, TIMP3 exerted an anti-apoptotic and anti-oxidative stress role in CIRI through the AKT pathway, which may be a potential therapeutic target for the treatment of CIRI.
\end{abstract}

Correspondence to: Professor Fengxiao Gao, Department of Imaging, Xingtai People's Hospital, 16 Hongxing Street, Xingtai, Hebei 054001, P.R. China

E-mail: gaofengxiao69@sina.com

Key words: cerebral ischemia-reperfusion injury, tissue inhibitor of metalloproteinases-3, AKT pathway, oxidative stress, TUNEL

\section{Introduction}

Stroke, also known as cerebral vascular accident, is characterized by sudden onset and the rapid occurrence of localized or diffuse brain dysfunction (1). Stroke includes ischemic stroke and hemorrhagic stroke, of which ischemic stroke accounts for $67.3-80.5 \%$ of cases, which seriously threatens human health and creates a large social burden (1). The main treatments for ischemic stroke are intravascular thrombolysis and mechanical thrombectomy, aiming to restore the blood supply of ischemic brain tissue through reperfusion (2). However, there is often an aggravation of tissue damage called cerebral ischemia-reperfusion injury (CIRI) when the blood flow is restored $(3,4)$. The mechanisms of CIRI include abnormal signal transduction, mitochondrial damage, oxidative stress, autophagy, inflammation and apoptosis $(5,6)$. Thus, avoiding the injury of CIRI is required in the early treatment of ischemic stroke.

Tissue inhibitor of metalloproteinases (TIMP)-3 is a member of the tissue inhibitors of metalloproteinases family, which prevent extracellular matrix degradation by inhibiting the activity of matrix metalloproteinases (MMPs) (7). Moreover, TIMP3 has been demonstrated to exert a role in regulating apoptosis in tumor cell lines and myocardial infarction, and also in inhibiting angiogenesis in addition to targeting MMPs (8-11). The overexpression of TIMP3 could attenuate oxidative stress in metabolic disorders (12). Similarly, TIMP3 also serves a notable role in ischemia-reperfusion (I/R) injury. A previous study has demonstrated that knockout of TIMP3 can aggravate the I/R injury of hepatocytes (13). By contrast, TIMP3-overexpression protects myocardial I/R injury by inhibiting cardiomyocyte apoptosis (11). However, the role of TIMP3 in CIRI remains unknown.

Due to the nerve growth factor (NGF) receptor on the cell membrane, PC12 cells can grow neurites and differentiate into neurons after being induced by NGF at the physiological level. Therefore, PC12 cells are widely used as in vitro model of cerebral ischemia injury $(14,15)$. In the current study, PC12 cells were used as a cell model of cerebral ischemia to study the effect of TIMP3 on oxygen glucose deprivation/reoxygenation (OGD/R)-induced PC12 cell injury. Additionally, numerous studies have indicated that the Akt pathway mediates the 
occurrence and development of cerebral ischemia injury, and AKT activity is significantly inhibited after cerebral ischemia damage (16,17). Meanwhile, TIMP3-mediated neuroprotection mainly depends on the activation of AKT signaling pathway (18). The present study aimed to investigate whether TIMP3 prevented cerebral ischemia/reperfusion and to explore the role of TIMP3.

\section{Materials and methods}

Animals. A total of 16 male C57BL/6 mice (8-10-weeks-old; weight, $20 \pm 2 \mathrm{~g}$ ) were purchased from the Model Animal Research Center of Nanjing University. The Ethics Committee of Xingtai People's Hospital (Xingtai, China) approved all animal experiments. All mice were kept in a climate-controlled room (temperature, $25 \pm 1^{\circ} \mathrm{C}$; relative humidity, $50-60 \%$; free access to food and water; $12 \mathrm{~h}$ light/dark cycle). Mice were randomly allocated to the sham group $(n=6)$ or the I/R group $(\mathrm{n}=10)$, including two mice that died after the transient middle cerebral artery occlusion (MCAO) with unknown exact cause. Animal health and behavior were monitored once a day before model construction, and once every $30 \mathrm{~min}$ after MCAO. Proper anesthetic procedures were used to ensure that the mice did not suffer unnecessarily during or after the experimental procedure.

Models. MCAO was conducted according to a previous study (19). In brief, the mice were anesthetized with an intraperitoneal injection of pentobarbital sodium $(60 \mathrm{mg} / \mathrm{kg})$ until loss of limb reflexes. Subsequently, unilateral MCAO was carried out by introducing a 4-0 nylon thread into the internal carotid artery after cutting the common carotid artery. The origin of the middle cerebral artery was occluded when a mild resistance was felt for $2 \mathrm{~h}$ followed by reperfusion. The mice in the sham group underwent the same surgery without occlusion. At $24 \mathrm{~h}$ after reperfusion, following sacrifice by anesthetic overload with intraperitoneal $500 \mathrm{mg} / \mathrm{kg}$ ketamine and $50 \mathrm{mg} / \mathrm{kg}$ xylazine (no breathing and heartbeat were used to verify mortality), the brain tissues were obtained for assays. The methods of euthanasia conformed to the American Veterinary Medical Association Guidelines for the Euthanasia of Animals, 2020 Edition (20).

TTC staining. The brain tissues were kept overnight at $-80^{\circ} \mathrm{C}$ and sliced into $2-\mathrm{mm}$ thick sections, which were cultured with $1 \%$ TTC for $15 \mathrm{~min}$ at $37^{\circ} \mathrm{C}$ to visualize the infarct area. The white area manifested infarcted brain tissues. Infarct area was measured with computer-assisted planimetry (ImageJ version 1.57; National Institutes of Health).

Immunohistochemistry. The brain tissues were fixed with $10 \%$ formalin at $4^{\circ} \mathrm{C}$ overnight and embedded in paraffin before being cut into $5-\mu \mathrm{m}$ sections. After that, the sections were deparaffinized with xylene, rehydrated in a graded alcohol series and heated until boiling in a microwave oven at $1200 \mathrm{w}$ for $5 \mathrm{~min}$ for antigen retrieval. Endogenous peroxidase activity was blocked with $3 \% \mathrm{H}_{2} \mathrm{O}_{2}$ at $37^{\circ} \mathrm{C}$ for $20 \mathrm{~min}$. Subsequently, these sections were treated with TIMP3 primary antibody (1:300; cat. no. 5673S; Cell Signaling Technology, Inc.). After incubation at $4^{\circ} \mathrm{C}$ for one night and washing with PBS, the sections were cultured with a HRP polymer-bound goat anti-rabbit secondary antibody (1:5,000; cat. no. ab7090; Abcam) at room temperature for $1 \mathrm{~h}$. These samples were then stained with 3,3-diaminobenzidine solution at room temperature for $10 \mathrm{~min}$ and hematoxylin at room temperature for $5 \mathrm{~min}$. Finally, the slides were observed using a light microscope and the positively stained cells were counted using GraphPad Prism 6 software (GraphPad Software, Inc.).

Induction of $O G D / R$ model. NGF-induced PC12 cells were obtained from the American Type Culture Collection and cultured in Dulbecco's Modified Eagle Medium (DMEM) with $10 \%$ fetal bovine serum (both from Gibco; Thermo Fisher Scientific, Inc.) at $37^{\circ} \mathrm{C}$ with $5 \% \mathrm{CO}_{2}$ for $24 \mathrm{~h}$. PC12 cells were then washed with glucose-free-Hanks' balanced salt solution (Gibco; Thermo Fisher Scientific, Inc.) twice and placed in glucose-free DMEM (Gibco; Thermo Fisher Scientific, Inc.) in a hypoxic incubator chamber $\left(1 \% \mathrm{O}_{2} ; 5 \% \mathrm{CO}_{2} ; 94 \% \mathrm{~N}_{2}\right)$ at $37^{\circ} \mathrm{C}$ for 2,4 and $8 \mathrm{~h}$. The cells were transferred to normal growth conditions (DMEM with $10 \%$ fetal bovine serum at $37^{\circ} \mathrm{C}$ with $5 \% \mathrm{CO}_{2}$ ) for $24 \mathrm{~h}$ for oxygen-glucose deprivation and reoxygenation (OGD/R) (21). PC12 cells without OGD/R treatment were used as the control.

Cell transfection. Recombinant adenovirus [human adenovirus (Ad) 5] of TIMP3 (Ad: TIMP3) and empty adenovirus (human adenovirus 5, Ad5) vector as control (Ad: Con) were purchased commercially from Hanbio Biotechnology Co., Ltd. PC12 cells were maintained at $37^{\circ} \mathrm{C}$ in a humidified atmosphere with $5 \%$ $\mathrm{CO}_{2}$. The cells were transfected with Ad: TIMP3 or Ad: Con $\left(100 \times 10^{8} \mathrm{PFU} / \mathrm{ml}\right)$ using polybrene (Sigma-Aldrich; Merck $\mathrm{KGaA}$ ) for $48 \mathrm{~h}$ and treated with or without AKT inhibitor AZD5363 (Selleck Chemicals) for another $48 \mathrm{~h}$. The cells were then subjected to the associated experiments.

Semi-quantitative $P C R$ and reverse transcriptionquantitative $(R T-q) P C R$ analysis. Total RNA from brain tissues and PC12 cells was extracted using TRIzol ${ }^{\circledR}$ reagent (Thermo Fisher Scientific, Inc.). The purity of RNA was used to detect the absorbance ratio of $260 / 280 \mathrm{~nm}$ by a NanoDrop ND-1000 spectrophotometer (Thermo Fisher Scientific, Inc.). Reverse transcription of RNA was performed using a PrimeScript RT kit (Takara Bio, Inc.). The temperature protocol used for RT was $37^{\circ} \mathrm{C}$ for $15 \mathrm{~min}$ and $85^{\circ} \mathrm{C}$ for $15 \mathrm{sec}$, then the sample was kept at $4^{\circ} \mathrm{C}$ for immediate use or $-20^{\circ} \mathrm{C}$ for long-term storage. RT-PCR was used to measure the expression of TIMP3 mRNA. The primers used in the study were as follows: TIMP3, forward 5'-GGCAACTGTGCTGAA CAGGAT-3', and reverse, 5'-GATGGCCAGCGTGACACT T-3'; 18s ribosomal RNA forward 5'-GTTGTGCTGACGGCG CA-3' and reverse, 5'-CCGGCTTCGTGGCAGCA-3'; $\beta$-actin, forward 5'-GTAAAGACCTCTATGCCAACA-3', and reverse, 5'-GGACTCATCGTACTCCTGCT-3'.

For semi-quantitative PCR, reactions used a PCR Master mix (cat. no. K0171; Thermo Fisher Scientific, Inc.) and were performed on a StepOnePlus system (Applied Biosystems; Thermo Fisher Scientific, Inc.), starting with denaturation at $94^{\circ} \mathrm{C}$ for $3 \mathrm{~min}$, followed by 35 cycles of $94^{\circ} \mathrm{C}$ for $30 \mathrm{sec}$, $55^{\circ} \mathrm{C}$ for $30 \mathrm{sec}$ and $72^{\circ} \mathrm{C}$ for $30 \mathrm{sec}$, with a final extension at $72^{\circ} \mathrm{C}$ for $7 \mathrm{~min}$. Amplified DNA fragments were analyzed by 
electrophoresis on $1 \%$ agarose gels with $5 \%$ ethidium bromide at $100 \mathrm{~V}$ for $30 \mathrm{~min}$ at room temperature, and these ethidium bromide stained DNA fragments were observed under UV illumination.

For RT-qPCR, the experiment was carried out using the Power SYBR ${ }^{\circledR}$ Green PCR Master mix (cat. no. 4309155; Thermo Fisher Scientific, Inc.) with ABI7300 detector (Applied Biosystems; Thermo Fisher Scientific, Inc.). The reaction parameters were as follows: $95^{\circ} \mathrm{C}$ For $10 \mathrm{~min}$, and 40 cycles at $95^{\circ} \mathrm{C}$ for $15 \mathrm{sec}$ and $60^{\circ} \mathrm{C}$ for $30 \mathrm{sec}$. The mRNA expression levels were quantified using the $2^{-\Delta \Delta \mathrm{Cq}}$ method (22) and normalized to the internal reference genes $\beta$-actin.

Western blotting. The brain tissues and PC12 cells were lysed with lysis buffer (Cell Signaling Technology, Inc.) at $4^{\circ} \mathrm{C}$ for $30 \mathrm{~min}$ before centrifuging $\left(4^{\circ} \mathrm{C} ; 14462 \mathrm{x} \mathrm{g} ; 5 \mathrm{~min}\right)$. The total proteins were in the supernatant. Concentrations of proteins extracted from cerebral tissues or PC12 cells were then determined using the BCA protein assay kit (Bio-Rad Laboratories, Inc.). After that proteins (10 $\mu \mathrm{g}$ per lane) were separated using a $10 \%$ SDS-PAGE gel, then they were transferred to PVDF membranes (MilliporeSigma) followed by blocking with 5\% non-fat milk (Bio-Rad Laboratories, Inc.) at room temperature for $1 \mathrm{~h}$. Next, the membrane was probed with the following primary antibodies: TIMP3 (1:1,000; cat. no. 5673S), Bax (1:1,000; cat. no. 5023S), Bcl-2 (1:1,000; cat. no. 15071S), caspase-3 (1:1,000; cat. no. 9662S), AKT Thr308 (1:500; cat. no. 13038S), AKT Ser473 (1:500; cat. no. 4060S), AKT (1:1,000; cat. no. 2920S), GAPDH $(1: 1,000$; cat. no. $5174 \mathrm{~S})$ and $\beta$-actin $(1: 2,000$; cat. no. 3700T) (all Cell Signaling Technology, Inc.). Afterwards, the membrane was cultured with HRP-conjugated IgG anti-mouse $(1: 2,000$; cat. no. 7076) or anti-rabbit secondary antibodies (1:2,000; cat. no. 7074) (both Cell Signaling Technology, Inc.) at room temperature for $2 \mathrm{~h}$ and washed with TBST $(0.05 \%$ Tween). Finally, the blots were analyzed using SuperSignal West Pico PLUS Chemiluminescent Substrate (Thermo Fisher Scientific, Inc.) and visualized via a Chemiluminescence Imaging system (ChemiScope 3600 Mini; Clinx Science Instruments Co., Ltd). The protein bands were quantified using GraphPad Prism 6 software (GraphPad Software, Inc.).

Detection of reactive oxygen species (ROS), superoxide dismutase (SOD) and malondialdehyde (MDA). The levels of ROS were detected using a ROS Assay kit (cat. no. S0033S; Beyotime Institute of Biotechnology), the level of total SOD was evaluated by Superoxide Dismutase (SOD) assay kit (cat.no. A001-3-2; Nanjing Jiancheng Bioengineering Institute), and the level of MDA was measured by Malondialdehyde (MDA) assay kit (cat. no. A003-1-2; Nanjing Jiancheng Bioengineering Institute). These kits were all used according to the manufacturers' instructions.

TUNEL assay. PC12 cells were fixed in PBS with $4 \%$ polyformaldehyde at room temperature for $15 \mathrm{~min}$. The cells were washed once with PBS and then, apoptosis of PC12 cells in the different groups was detected by TUNEL via a in situ Cell Death Detection kit (Roche Applied Science). The kit was used in accordance with the manufacturer's protocol, and the ratio of apoptotic to total cells was calculated under fluorescence microscopy by observing three fields of view (magnification, $\mathrm{x} 400$ ). After that, ImageJ software (version 1.53, National Institutes of Health) was used to quantify the apoptosis rate.

Statistical analysis. SPSS version 20.0 statistic software (IBM Corp.) was used to analyze the data, which was expressed as mean \pm SD (unless otherwise presented). Statistical analysis was carried out using unpaired Student's t-test between two groups. Comparisons among two or more groups were analyzed using one-way ANOVA followed by Turkey's multiple comparisons post hoc test. $\mathrm{P}<0.05$ was considered to indicate a statistically significant difference.

\section{Results}

TIMP3 expression is downregulated in OGD/R-induced PC12 cells and cerebral tissues after I/R injury. To investigate the expression difference of TIMP3 after cerebral I/R injury in vivo, a model of cerebral I/R injury was constructed in mice. The morphology of the cerebral I/R injury model is presented in Fig. 1A. As demonstrated in Fig. 1B and C, the expression levels of TIMP3 in I/R-induced injury in mice cerebral tissues were significantly downregulated compared with the sham group. Moreover, immunohistochemical results indicated that the expression of TIMP3-positive cells decreased significantly in the I/R group (Fig. 1D). RT-qPCR and western blotting results indicated that the TIMP3 expression in OGD/R-induced PC12 cells was significantly downregulated in a time-dependent manner (Fig. 1E and F). These results demonstrated that TIMP3 expression was downregulated in $\mathrm{I} / \mathrm{R}$ injury in vivo and in vitro.

TIMP3 inhibits OGD/R-induced PC12 apoptosis and oxidative stress. PC12 cells were transfected with Ad-TIMP3 before OGD $8 \mathrm{~h} / \mathrm{R}$ treatment to confirm whether overexpression of TIMP3 could protect neurocytes from OGD/R-induced apoptosis and oxidative stress. The expression of TIMP3 was markedly overexpressed by Ad-TIMP3 therapy for $48 \mathrm{~h}$ (Fig. 2A). The number of TUNEL-positive cells in OGD/R-induced cells significantly increased after OGD/R, but was significantly reduced by TIMP3-overexpression (Fig. 2B). Western blotting indicated that OGD/R increased the $\mathrm{Bax} / \mathrm{Bcl}-2$ ratio, and caspase-3 expression compared with the NC; by contrast, this was significantly attenuated by TIMP3-overexpression (Fig. 2C). In addition, the levels of ROS and MDA were significantly elevated in OGD/R-induced PC12 cells compared with control cells, while this was significantly suppressed by TIMP3-overexpression. Conversely, SOD activity decreased significantly in PC12 cells after OGD/R treatment, and overexpression of TIMP3 significantly elevated SOD activity in the OGD/R-induced neurocytes (Fig. 2D). The data revealed that TIMP3 inhibited apoptosis and oxidative stress of OGD/R-induced PC12.

TIMP3 regulates the expression of phosphorylated $(p)-A K T$. As TIMP3 was demonstrated to exert its function by regulating the AKT pathway, the phosphorylation levels of AKT (Thr308 and Ser473) were detected. Western blotting demonstrated that p-AKT (Thr308 and Ser473) was significantly downregulated in OGD/R-treated PC12 cells, which could be 
A

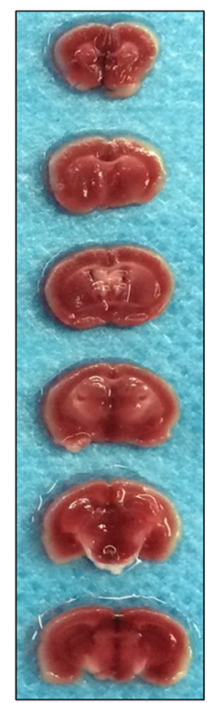

Sham

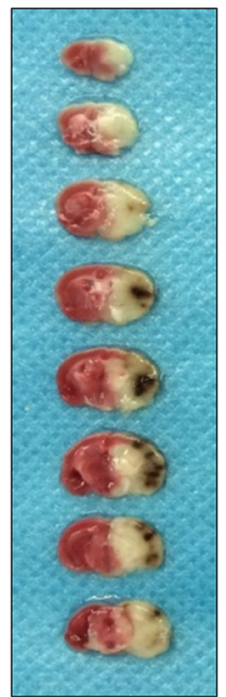

I/R
B

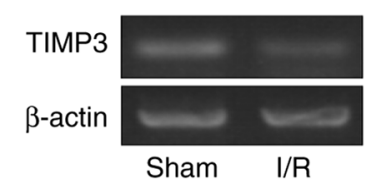

C

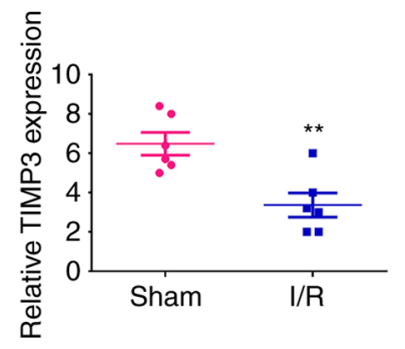

D

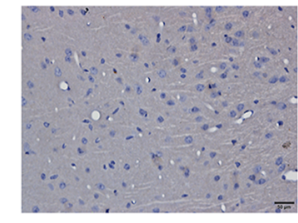

Sham

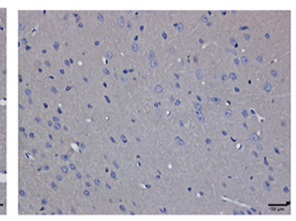

I/R
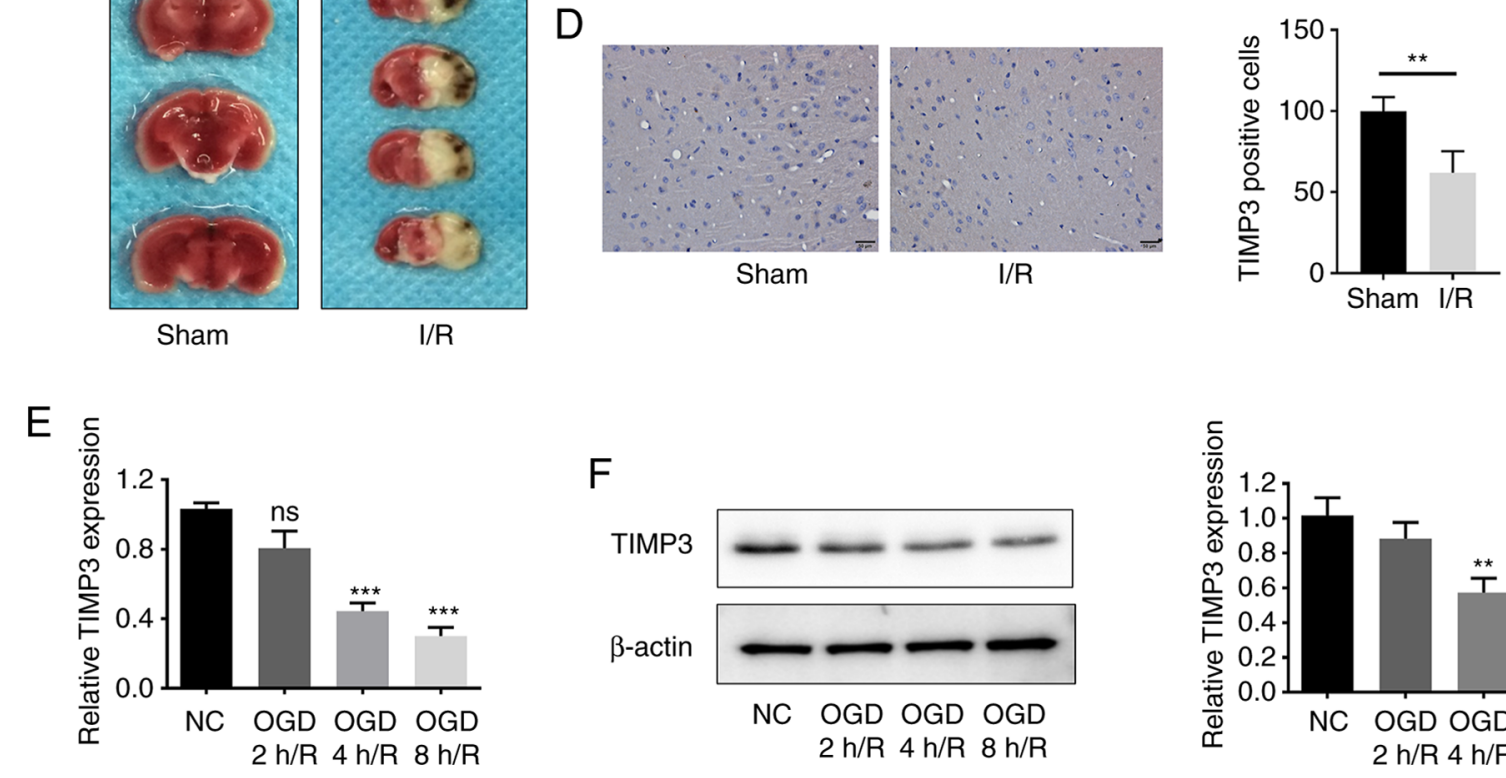

$\mathrm{F}$

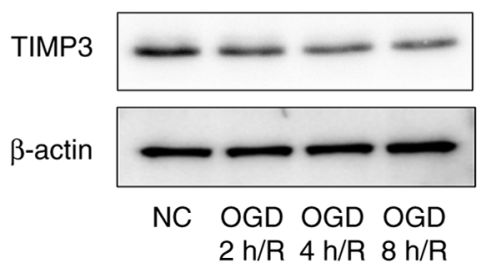

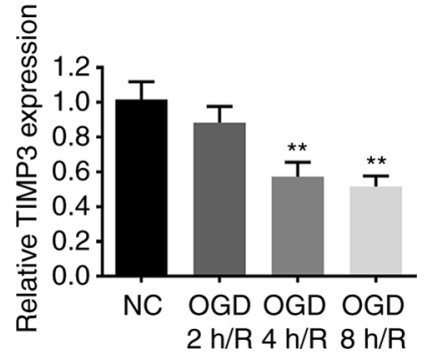

Figure 1. TIMP3 expression is downregulated in OGD/R-induced PC12 cells and cerebral tissues after I/R injury. (A) Model mice of cerebral I/R injury and the control group received TTC staining. (B and C) Semi quantitative RT-PCR was used to detect the expression of TIMP3 after I/R injury in mouse cerebral tissue. (D) Immunohistochemical staining was used to evaluate the TIMP3-positive cells between sham and I/R groups. (E) Expression levels of TIMP3 in cerebral tissues after I/R injury was detected by RT-quantitative PCR analysis. (F) Western blotting of TIMP3 expression in OGD/R-induced PC1 2 cells. ${ }^{* *} \mathrm{P}<0.01$ and ${ }^{* * *} \mathrm{P}<0.001$ vs. sham or NC. TIMP3, tissue inhibitor of metalloproteinases-3; OGD/R, oxygen glucose deprivation and reoxygenation; $\mathrm{I} / \mathrm{R}$, ischemia-reperfusion; RT, reverse transcription; ns, no significance; $\mathrm{NC}$, negative control.

significantly reversed by TIMP3-overexpression. However, TIMP3-overexpression did not significantly affect p-AKT expression in cells without OGD/R treatment (Fig. 3A). In addition, in order to further conduct the rescue experiment in the following mechanism study, the p-AKT inhibitor (AZD5363) was used to inhibit p-AKT (Thr308 and Ser473) activity (Fig. 3B). The experiments demonstrated that the expression of p-AKT (Thr308 and Ser473) was regulated by TIMP3.

Overexpression of TIMP3 inhibits OGD/R-induced PC12 apoptosis and oxidative stress via the AKT pathway. After confirming that TIMP 3 could elevate the expression levels of p-AKT (Thr308 and Ser473) that had been decreased by OGD/R treatment, whether the function of TIMP3 could be mediated via p-AKT overexpression was investigated. TIMP3-overexpression significantly reduced the number of TUNEL-positive cells that were elevated in OGD/R-induced neurocytes compared with the $\mathrm{OGD} / \mathrm{R}+\mathrm{Ad}$-control group. This effect of TIMP3 in OGD/R-induced PC12 cells was partially but significantly reversed after AZD5363 treatment (Fig. 4A). Western blotting indicated that TIMP3 downregulated the expression of Bax and upregulated the expression of Bcl-2 in OGD/R, which were partially reversed after AZD5363 treatment (Fig. 4B). Consistent with the aforementioned results, the anti-oxidative stress role of TIMP3 in OGD/R-induced PC12 cells was also partially blocked by AZD5363 treatment (Fig. 4C). Thus, it is confirmed that TIMP3 inhibits apoptosis and oxidative stress via AKT pathway in vitro.

\section{Discussion}

CIRI is one of the important factors that aggravates brain injury after cerebral ischemia. Reducing reperfusion injury helps protect neurons in the ischemic area and reduces neuron necrosis and apoptosis (2). Various mechanisms have been demonstrated to be involved in the development of CIRI, including apoptosis and oxidative stress (3). Thus, treatments that improve apoptosis and oxidative stress may have potential therapeutic effects for CIRI.

TIMP3 is a member of the TIMP family of proteins, which are characterized as inhibitors of MMPs (7). However, with increasing research, the role of TIMP 3 in regulating apoptosis and alleviating oxidative stress has attracted much attention $(11,12)$. For example, Liu et al (11) revealed that TIMP3 upregulation could protect against cardiac I/R injury by inhibiting myocardial apoptosis. In the present study, the expression 

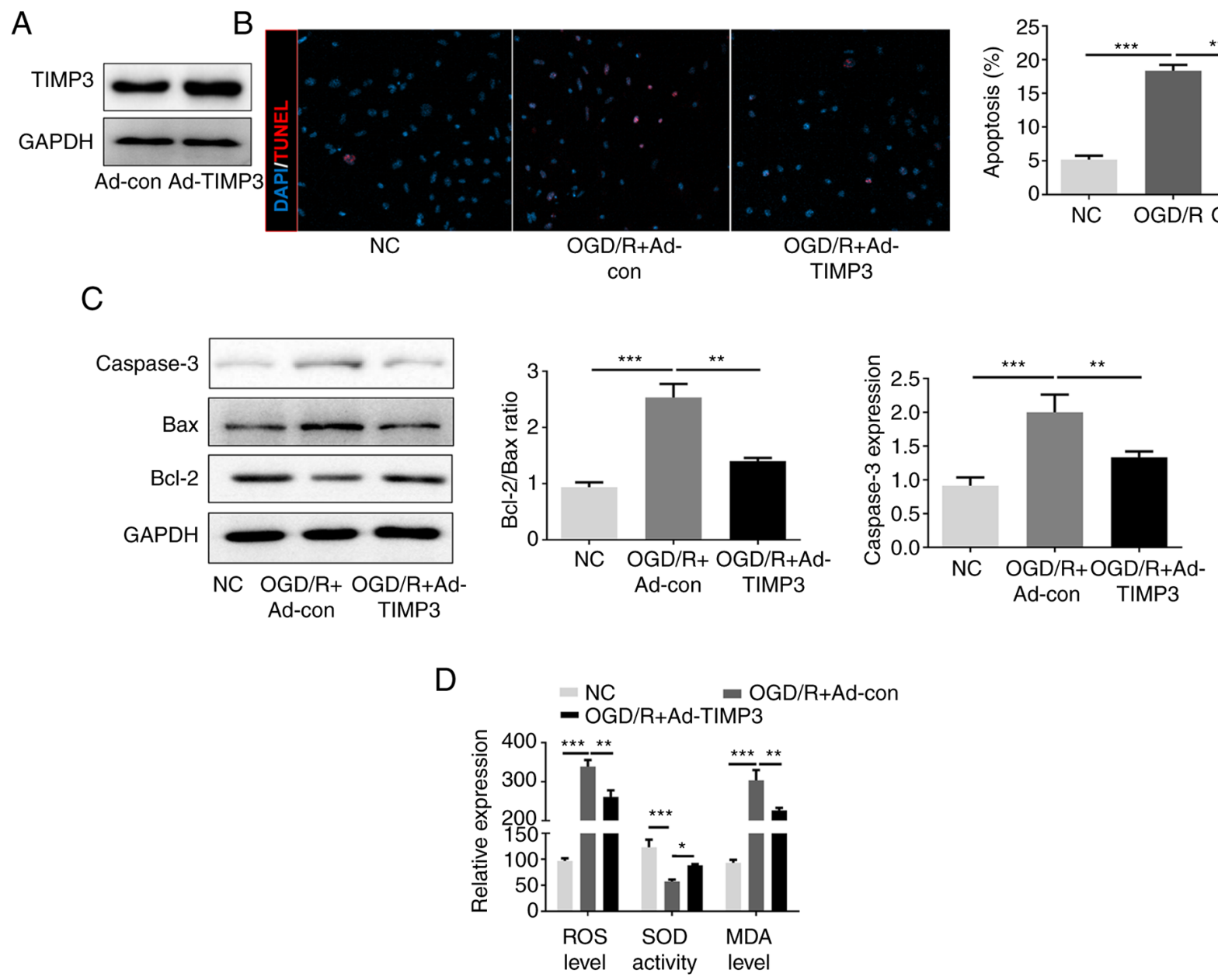

Figure 2. TIMP3 inhibits OGD/R-induced PC12 apoptosis and oxidative stress. (A) Western blotting was used to confirm the expression of TIMP3 by Ad-TIMP3. (B) TUNEL immunofluorescence staining was used to examine apoptosis-positive cells in different groups (magnification, $\mathrm{x} 400$ ). (C) Western blotting was performed to detect the expression of apoptosis proteins, Bax and Bcl-2. (D) ROS generation, MDA level and SOD activity were detected after different treatments using corresponding commercial kits. ${ }^{*} \mathrm{P}<0.05,{ }^{* *} \mathrm{P}<0.01$ and ${ }^{* * * *} \mathrm{P}<0.001$ as indicated. TIMP3, tissue inhibitor of metalloproteinases-3; OGD/R, oxygen glucose deprivation and reoxygenation; Ad, adenovirus; ROS, reactive oxygen species; MDA, malondialdehyde; SOD, superoxide dismutase; $\mathrm{NC}$, negative control; con, control.
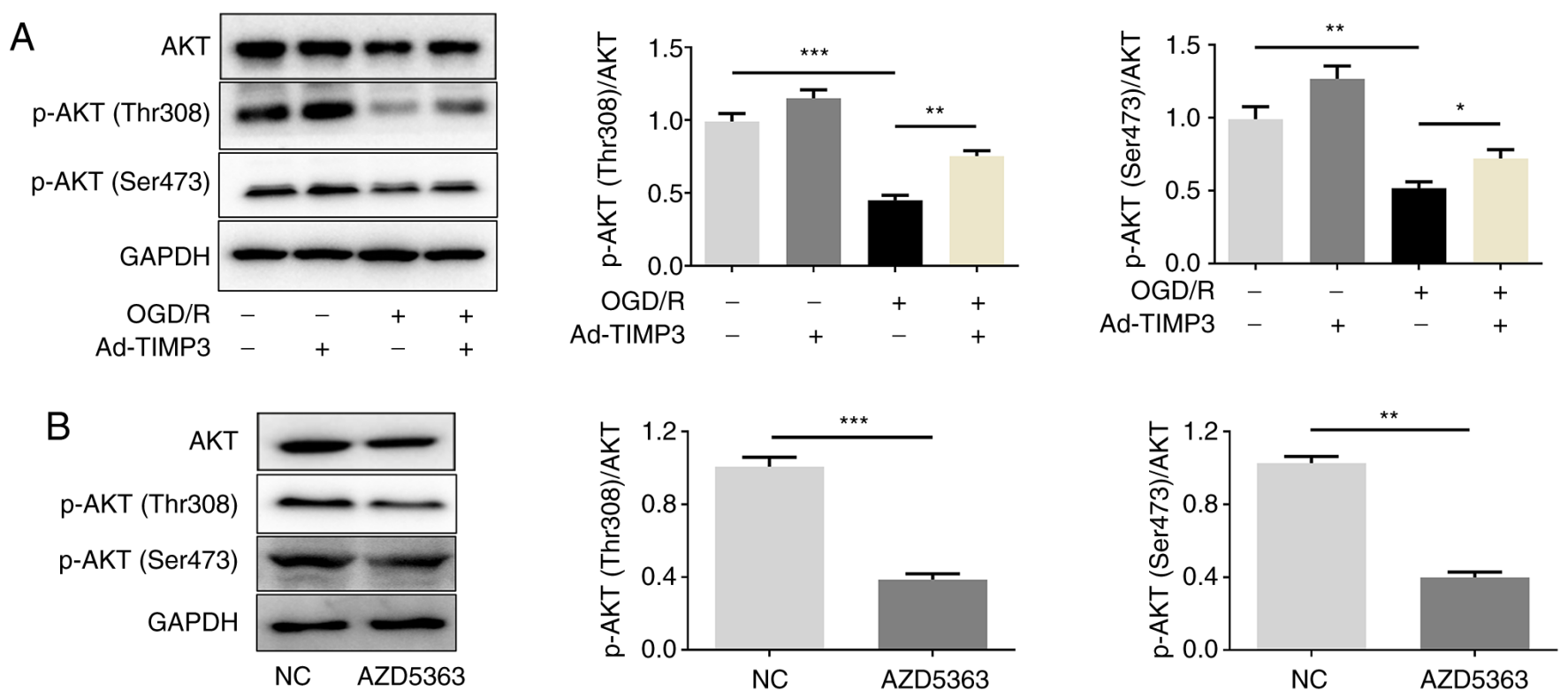

Figure 3. TIMP3 regulates the expression of phosphorylated AKT. Western blotting was performed and quantified to examine AKT and p-AKT (Thr308 and Ser473) protein expression levels after (A) transfection with Ad-TIMP3 or (B) treatment with p-AKT inhibitor (AZD5363). ${ }^{*} \mathrm{P}<0.05,{ }^{* *} \mathrm{P}<0.01$ and ${ }^{* * * *} \mathrm{P}<0.001$ as indicated. TIMP3, tissue inhibitor of metalloproteinases-3; p, phosphorylated; Ad, adenovirus; OGD/R, oxygen glucose deprivation and reoxygenation. 


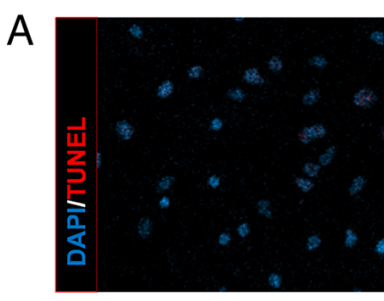

NC

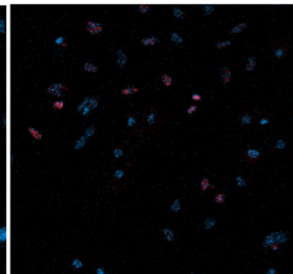

OGD/R+Ad-

con

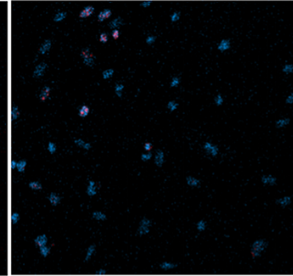

$\mathrm{OGD} / \mathrm{R}+\mathrm{Ad}-$

TIMP3

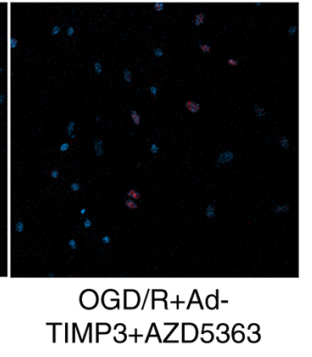

TIMP3+AZD5363

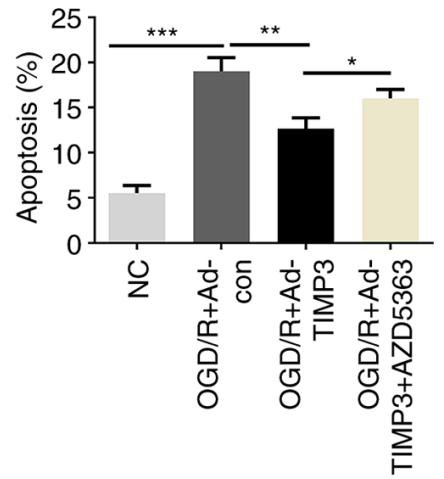

B
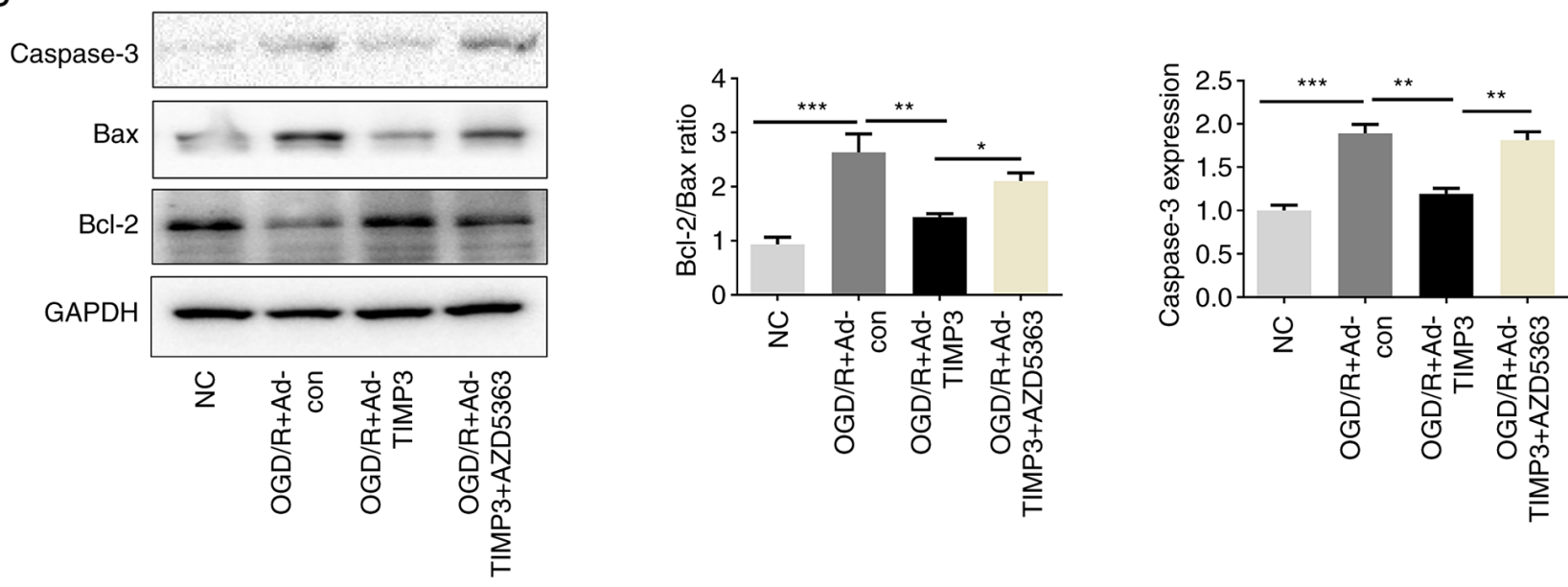

C

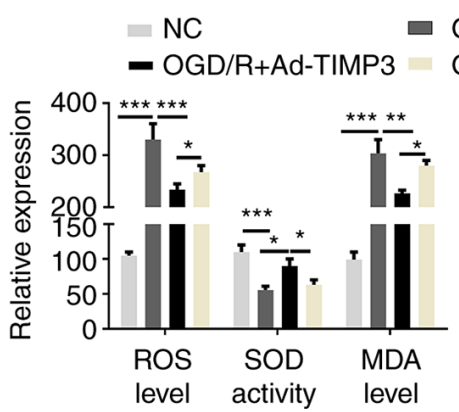

Figure 4. TIMP3 inhibits OGD/R-induced PC12 apoptosis and oxidative stress via the AKT pathway. (A) TUNEL immunofluorescence staining was used and quantified to detect the apoptosis-positive cells in different groups (magnification, x400). (B) Western blotting was conducted to determine the expression levels of apoptosis proteins Bax and Bcl-2. (C) ROS generation, MDA level and SOD activity after different treatments were detected by corresponding commercial kits. ${ }^{*} \mathrm{P}<0.05,{ }^{* *} \mathrm{P}<0.01$ and ${ }^{* * *} \mathrm{P}<0.001$ as indicated. TIMP3, tissue inhibitor of metalloproteinases-3; OGD/R, oxygen glucose deprivation and reoxygenation; ROS, reactive oxygen species; MDA, malondialdehyde; SOD, superoxide dismutase; con, control; Ad, adenovirus; NC, negative control.

of TIMP3 was significantly decreased in the cerebral tissues after I/R injury and in OGD/R-induced PC12 cells. The results indicated that TIMP3 may have a pathological role in CIRI.

Mitochondrial dysfunction and oxidative stress are responsible for neuronal damage, which eventually leads to a cascade of apoptosis (23). Brain injury is associated with an increase of apoptosis, which is characterized by the increased expression of Bax and the decreased expression of Bcl-2; meanwhile, inhibition of apoptosis has been demonstrated to attenuate neuronal damage in CIRI (24). The overproduction of ROS and the weakening of the antioxidant mechanism are notable pathological events in the process of CIRI (25). The increase of ROS leads to the oxidation of lipids, proteins and nucleic acids, which changes the function of cells (26). MDA is one of the essential products of membrane lipid peroxidation, thus, its expression reflects the degree of oxidative stress (26). Therefore, the clearance of ROS is particularly important in CIRI. SOD has a significant scavenging effect on ROS produced in the process of oxidation (26). In the present study, overexpression of TIMP3 reduced the number of TUNEL-positive cells and attenuated the Bax/Bcl-2 ratio in OGD/R-induced PC12 cells. In addition, overexpression of TIMP3 could suppress the levels of ROS and MDA, and also elevate SOD activity in the OGD/R-induced neurocytes. These data indicated that overexpression of TIMP3 could protect neurocytes from OGD/R-induced apoptosis and oxidative stress in vitro. The effect of TIMP3 in cerebral tissues after I/R injury in vivo would need to be further investigated to confirm these results.

The AKT pathway serves a notable role in regulating CIRI (27); TIMP3 exerts its function by regulating the AKT pathway (18). The results of the current study indicated that TIMP3-overexpression could reverse the downregulation of 
p-AKT (Thr308 and Ser473) in OGD/R-treated PC12 cells. Further rescue studies indicated that the anti-apoptosis and anti-oxidative stress role of TIMP3 in OGD/R-induced PC12 cells could be partially abolished after AZD5363 treatment. This demonstrated that TIMP3 attenuated CIRI apoptosis and oxidative stress in neurocytes by regulating the AKT pathway. However, the specific binding molecules and their sites of binding to TIMP3 to modify the phosphorylation of AKT in I/R injury were unknown, which will be investigated further in the future. It has been previously demonstrated that the AKT pathway is activated in hypoxia-induced neuronal injury, which is contrary to the findings of the present study (28). These inconsistent results may be caused by the different models applied. Continuous lack of glucose oxygen was possibly a factor in AKT phosphorylation. Thus, the opposite effect on AKT activity would be observed under glucose and oxygen-glucose deprivation condition.

In conclusion, the present study demonstrated that TIMP3 exerted anti-apoptotic and anti-oxidative stress roles in cerebral I/R injury and provided a potential novel therapeutic target for the treatment of CIRI.

\section{Acknowledgments}

Not applicable.

\section{Funding}

The work was supported by The Xingtai Science and Technology Support Plan Project (grant no. 2018ZC191).

\section{Availability of data and materials}

The datasets used and/or analyzed during the current study are available from the corresponding author on reasonable request.

\section{Authors' contributions}

LM, YZ and FG proposed the project, aims and objectives. LM, YZ, DL and XS conducted the experiments and collected the data. LM, YZ, XH, XC and FG analyzed the results and wrote the manuscript. FG submitted the manuscript. LM and FG confirm the authenticity of all the raw data. All authors have read and approved the final manuscript.

\section{Ethics approval and consent to participate}

All animal experiments were conducted according to the ethical guidelines of The Xingtai People's Hospital and the '3R' principle (Replacement, Reduction, and Refinement). All efforts were made to minimize animal suffering. The present study was approved by The Ethics Committee of Xingtai People's Hospital (Xingtai, China).

\section{Patient consent for publication}

Not applicable.

\section{Competing interests}

The authors declare that they have no competing interests.

\section{References}

1. Bosetti F, Koenig JI, Ayata C, Back SA, Becker K, Broderick JP, Carmichael ST, Cho S, Cipolla MJ, Corbett D, et al: Translational stroke research: Vision and opportunities. Stroke 48: 2632-2637, 2017.

2. Dorado L, Millan M and Davalos A: Reperfusion therapies for acute ischemic stroke: An update. Curr Cardiol Rev 10: 327-335, 2014.

3. Nishijima Y, Akamatsu Y, Weinstein PR and Liu J: Collaterals: Implications in cerebral ischemic diseases and therapeutic interventions. Brain Res 1623: 18-29, 2015.

4. Zhang J, Fang X, Zhou Y, Deng X, Lu Y, Li J, Li S, Wang B and $\mathrm{Xu} \mathrm{R}$ : The possible damaged mechanism and the preventive effect of monosialotetrahexosylganglioside in a rat model of cerebral ischemia-reperfusion injury. J Stroke Cerebrovasc Dis 24: 1471-1478, 2015.

5. Liang G, Shi B, Luo W and Yang J: The protective effect of caffeic acid on global cerebral ischemia-reperfusion injury in rats. Behav Brain Funct 11: 18, 2015.

6. Siroli L, Braschi G, de Jong A, Kok J, Patrignani F and Lanciotti R: Transcriptomic approach and membrane fatty acid analysis to study the response mechanisms of Escherichia coli to thyme essential oil, carvacrol, 2-(E)-hexanal and citral exposure. J Appl Microbiol 125: 1308-1320, 2018.

7. Di Gregoli K, Mohamad Anuar NN, Bianco R, White SJ, Newby AC, George SJ and Johnson JL: MicroRNA-181b controls atherosclerosis and aneurysms through regulation of TIMP-3 and elastin. Circ Res 120: 49-65, 2017.

8. Brew $\mathrm{K}$ and Nagase H: The tissue inhibitors of metalloproteinases (TIMPs): An ancient family with structural and functional diversity. Biochim Biophys Acta 1803: 55-71, 2010.

9. Shen B, Jiang Y, Chen YR, Zheng HC, Zeng W, Li YY, Yin A and Nie Y: Expression and inhibitory role of TIMP-3 in hepatocellular carcinoma. Oncol Rep 36: 494-502, 2016.

10. Kubatka P, Uramova S, Kello M, Kajo K, Kruzliak P, Mojzis J, Vybohova D, Adamkov M, Jasek K, Lasabova Z, et al: Antineoplastic effects of clove buds (Syzygium aromaticum L.) in the model of breast carcinoma. J Cell Mol Med 21: 2837-2851, 2017.

11. Liu H, Jing X, Dong A, Bai B and Wang H: Overexpression of TIMP3 protects against cardiac ischemia/reperfusion injury by inhibiting myocardial apoptosis through ROS/Mapks pathway. Cell Physiol Biochem 44: 1011-1023, 2017.

12. Menghini R, Casagrande V, Menini S, Marino A, Marzano V, Hribal ML, Gentileschi P, Lauro D, Schillaci O, Pugliese G, et al: TIMP3 overexpression in macrophages protects from insulin resistance, adipose inflammation, and nonalcoholic fatty liver disease in mice. Diabetes 61: 454-462, 2012.

13. Fujii T, Duarte S, Lee E, Ke B, Busuttil RW and Coito AJ: Tissue inhibitor of metalloproteinase 3 deficiency disrupts the hepatocyte E-cadherin/ $\beta$-catenin complex and induces cell death in liver ischemia/reperfusion injury. Liver Transpl 26: 113-126, 2020.

14. Wang G, Wang T, Hu Y, Wang J, Wang Y, Zhang Y, Li F, Liu W, Sun Y, Yu B and Kou J: NMMHC IIA triggers neuronal autophagic cell death by promoting F-actin-dependent ATG9A trafficking in cerebral ischemia/reperfusion. Cell Death Dis 11: $428,2020$.

15. Zhou Z, Xu N, Matei N, McBride DW, Ding Y, Liang H, Tang J and Zhang JH: Sodium butyrate attenuated neuronal apoptosis via GPR41/G $\beta \gamma / \mathrm{PI} 3 \mathrm{~K} / \mathrm{Akt}$ pathway after MCAO in rats. J Cereb Blood Flow Metab 41: 267-281, 2021.

16. Li Y, Guo S, Liu W, Jin T, Li X, He X, Zhang X, Su H, Zhang N and Duan C: Silencing of SNHG12 enhanced the effectiveness of MSCs in alleviating ischemia/reperfusion injuries via the PI3K/AKT/mTOR signaling pathway. Front Neurosci 13: 645, 2019.

17. Feng H, Hu L, Zhu H, Tao L, Wu L, Zhao Q, Gao Y, Gong Q, Mao F, Li X, et al: Repurposing antimycotic ciclopirox olamine as a promising anti-ischemic stroke agent. Acta Pharm Sin B 10: 434-446, 2020.

18. Gibb SL, Zhao Y, Potter D, Hylin MJ, Bruhn R, Baimukanova G, Zhao J, Xue H, Abdel-Mohsen M, Pillai SK, et al: TIMP3 attenuates the loss of neural stem cells, mature neurons and neurocognitive dysfunction in traumatic brain injury. Stem Cells 33: 3530-3544, 2015.

19. Bu X, Zhang N, Yang X, Liu Y, Du J, Liang J, Xu Q and Li J: Proteomic analysis of cPKC $\beta$ II-interacting proteins involved in $\mathrm{HPC}$-induced neuroprotection against cerebral ischemia of mice. J Neurochem 117: 346-356, 2011. 
20. Leary S, Anthony R, Grandin T, Greenacre C, Gwaltney-Brant S, Ann McCrackin M, Meyer R, Miller D, Shearer J, Turner T and Yanong R: AVMA guidelines for the euthanasia of animals: 2020 Edition*. AVMA, 2020.

21. Liu X, Li M, Hou M, Huang W and Song J: MicroRNA-135a alleviates oxygen-glucose deprivation and reoxygenation-induced injury in neurons through regulation of GSK-3 $/ \mathrm{Nrf} 2$ signaling. J Biochem Mol Toxicol: e22159, 2018. doi: 10.1002/jbt.22159.

22. Livak KJ and Schmittgen TD: Analysis of relative gene expression data using real-time quantitative PCR and the 2(-Delta Delta C(T)) method. Methods 25: 402-408, 2001.

23. Broughton BR, Reutens DC and Sobey CG: Apoptotic mechanisms after cerebral ischemia. Stroke 40: e331-e339, 2009.

24. Li P, Shen M, Gao F, Wu J, Zhang J, Teng F and Zhang C: An antagomir to microRNA-106b-5p ameliorates cerebral ischemia and reperfusion injury in rats via inhibiting apoptosis and oxidative stress. Mol Neurobiol 54: 2901-2921, 2017.
25. Woodruff TM, Thundyil J, Tang SC, Sobey CG, Taylor SM and Arumugam TV: Pathophysiology, treatment, and animal and cellular models of human ischemic stroke. Mol Neurodegener 6: 11, 2011.

26. Lewen A, Matz P and Chan PH: Free radical pathways in CNS injury. J Neurotrauma 17: 871-890, 2000.

27. Liu X, Qing Wang, Cui Y, Li X and Yang H: In-depth transcriptomic and proteomic analyses of the hippocampus and cortex in a rat model after cerebral ischemic injury and repair by Shuxuetong (SXT) injection. J Ethnopharmacol 249: 112362, 2019.

28. Barialai L, Strecker MI, Luger AL, Jäger M, Bruns I, Sittig ACM, Mildenberger IC, Heller SM, Delaidelli A, Lorenz NI, et al: AMPK activation protects astrocytes from hypoxia-induced cell death. Int J Mol Med 45: 1385-1396, 2020.

cc) (i) $(-)$ This work is licensed under a Creative Commons CY NC ND Attribution-NonCommercial-NoDerivatives 4.0 International (CC BY-NC-ND 4.0) License. 